\title{
Perfil Audiológico de Pilotos Agrícolas
}

\section{Agricultural Pilot's Audiological Profile}

\section{Lucas Foltz*, Carla Debus Soares**, Maria Adelaide Kubl Reichembach***.}

\author{
*Bacharel. Fonoaudiólogo. \\ ** Mestrado. Professora do Curso de Fonoaudiologia do Centro Universitário Metodista IPA e Fonoaudióloga Clínica do Mãe de Deus Center e Hopital Mãe de Deus \\ de Porto Alegre / RS. \\ *** Especialista em Voz - CEFAC - POA. Fonoaudióloga militar (Tenente Coronel) - Chefe da Seção de Fonoaudiologia e chefe de Divisão de Ensino e Pesquisa do \\ HACO - RS. \\ Instituição: Centro Universitário Metodista IPA \\ Porto Alegre / RS - Brasil. \\ Endereço para correspondência: Lucas Foltz - Rua Alexandre da Motta, 943 - Apto 308 -Bairro Centro - Carazinho / RS - Brasil - CEP: $99500-000$ - Telefone: \\ (+55 54) 8401-2628 - E-mail: fono.lucasfoltz@hotmail.com \\ Artigo recebido em 15 de Abril de 2010. Artigo aprovado em 20 de Maio de 2010.
}

\section{RESUMO}

Introdução:

Objetivo:

Método:

Resultados:

Conclusão:

Palavras-chave:

\section{SUMMARY}

Introduction:

Objective:

Method:

Results:

Conclusion:

Keywords:
Os pilotos de aviões agrícolas estão diariamente expostos a ruídos intensos, estando suscetíveis à Perda Auditiva Induzida pelo Ruído (PAIR) e seus efeitos auditivos e extra-auditivos.

Analisar o perfil audiológico desta população, verificando a influência do trabalho sobre sua audição. Foi realizado um estudo retrospectivo, de corte transversal, individual e observacional, através de dados obtidos por meio de questionário e audiometria tonal liminar de 41 pilotos agrícolas. Para a análise estatística foram utilizados os testes Qui-quadrado, Spearman e Wilcoxon, com nível de significância de $5 \%$.

Verificou-se que $95,1 \%$ dos pilotos usam EPI durante os vôos e 58,5\% possuem contato com agrotóxicos. Mais da metade dos indivíduos referiram ter sintomas auditivos e extra-auditivos, sendo o zumbido o mais frequente (29,1\%). Houve a ocorrência de 29,3\% de perda auditiva sugestiva de PAIR e 68,3\% de normalidade, tendo, esta, presença de entalhe unilateral em 24,4\% e entalhe bilateral em 31,7\%. Foi encontrada correlação estatisticamente significativa nas associações entre tempo de serviço e a média das frequências agudas na orelha direita $(p=0038)$ e na orelha esquerda $(p=0,010)$. Houve tendência estatística na associação entre configuração audiométrica e contato com agrotóxicos ( $\mathrm{p}=0,088)$.

A prevalência da perda auditiva neste estudo se mostrou elevada. Mais da metade da amostra possui limiares auditivos normais com configuração de entalhe. Tais dados levam a conclusão de que os pilotos agrícolas, mesmo com o uso de EPI, ainda sofrem com os danos provocados pelo ruído, carecendo de melhores propostas de prevenção da perda auditiva.

perda auditiva provocada por ruído, aviação, agricultura, ruído ocupacional, saúde do trabalhador.

The agricultural airplane pilot are daily exposed to intense noises, being susceptible to the noiseinduced hearing loss (NIHL) and its auditory and extra auditory effects.

To analyze the audiological profile of this population, verifying the work's influence on its hearing. It was realized a retrospective, individual, observational, and cross-sectional study through the data obtained by means of a questionnaire and audiometric thresholds of 41 agricultural pilots. To the statistical analysis were utilized the chi-square, Spearman, and Wilcoxon tests with significance level of $5 \%$.

It was verified that $95,1 \%$ of the pilots use PPE ( personal protective equipment) during flight and 58,5\% have contact with pesticides. More than half of individuals referred to feel auditory and extra auditory symptoms, being the buzz the more frequent (29,1\%). It has the occurrence of $29,3 \%$ of NIHL suggestive hearing loss and $68,3 \%$ of normality, taking this presence of unilateral notch in $24,4 \%$ and bilateral notch in $31,7 \%$. It was found correlation statistically significant in the associations between time of service and the average of the acute frequencies in the right ear $(\mathrm{p}=0038)$, and in the left ear $(\mathrm{p}=0,010)$. It has a statistical tendency in the association between audiometric configuration and contact with pesticides $(\mathrm{p}=0,088)$.

The hearing loss prevalence in this study was showed high. More than half of the sample has normal audiometric thresholds with notch configuration. Such data lead to the conclusion that the agricultural pilots, even with PPE use, they still suffer with the damages caused by noise, needing best proposals of hearing loss prevention.

noise-induced hearing loss, aviation, agriculture, occupational noise, worker health. 


\section{INTRODUÇÃO}

A aviação agrícola chegou ao Brasil pela necessidade de controle de pragas emáreas extensas de monocultura, já que os sistemas mecanizados de aplicação de defensivos agrícolas terrestres eram muito lentos e não conseguiam controlar efetivamente toda a plantação. Atualmente, a aviação agrícola tem como atividades a aplicação de fertilizantes, defensivos agrícolas, semeadura, povoamento de águas, combate a incêndios e a vetores (1).

Os aviões agrícolas possuem a característica de serem, em sua maioria, monomotores, tendo a localização do motor na parte frontal e acomodando apenas um tripulante. Dessa forma, o ruído produzido pelo motor fica próximo da cabine do piloto, chegando ao cockpit em intensidades elevadas.

O ambiente de aeroportos e aeroclubes possui múltiplas fontes de ruído, tanto em terra quanto no ar. O ruído é produzido pelos equipamentos de força da aeronave, sistemas de transmissão, hélices, rotores, atuadores hidráulicos e elétricos, ar condicionado da cabine e sistemas de pressurização, sistema de alertas e equipamentos de comunicações. O ruído pode também ser gerado pela interação aerodinâmica entre o ar (camada limite) e a superfície da aeronave, como fuselagem, asas, superfície de controle e trem de pouso. Estes sons não apenas tornam o ambiente de trabalho mais estressante, mas podem ao longo do tempo causar alterações auditivas (2).

Gerostergiou et al (3) realizou um estudo envolvendo 15 pilotos de um aeroclube, sendo feitas as avaliações audiológicas e do nível do ruído de aviões de pequeno porte e ultra-leves. Os achados demonstraram que $30 \%$ dos aviadores possuíam alteração auditiva sugestiva de Perda Auditiva Induzida pelo Ruído (PAIR) e o nível de ruído atingiu o pico de 100-110dB, com média de $75 \mathrm{~dB}$ em ambos os modelos.

As alterações ou efeitos que o ruído acarreta à audição e à saúde, em geral, são influenciados pelos níveis de pressão sonora (NPS), pelo tipo de ruído, pela frequência, pelo tempo total de exposição e ainda pela suscetibilidade individual. Dessa forma, o ruído pode afetar os sujeitos expostos de formas diferentes pelo mesmo período de tempo (4).

Vários estudos, voltados à PAIR, mostraram correlação entre os limiares audiométricos e os anos de profissão $(5,6,7)$.

O trauma acústico, a mudança temporária do limiar (MTLA) e a PAIR são caracterizadas como alterações auditivas decorrentes da exposição ao ruído. O trauma acústico caracteriza-se pela exposição única a NPS muito elevados, resultando em uma lesão permanente e imediata, podendo ser unilateral ou bilateral. A MTLA refere-se a uma dificuldade auditiva ocasionada por exposição curta a NPS elevados, podendo estar acompanhada de zumbido. Após o repouso acústico os limiares retornam ao seu padrão inicial. Já a PAIR é definida como a perda de audição permanente procedida pela exposição prolongada e repetida a níveis de ruído intensos (8).

A Portaria no 19 do Ministério do Trabalho (9) estabelece que a PAIR é determinada pelas alterações dos limiares auditivos do tipo neurossensorial, decorrente da exposição ocupacional sistemática a NPS elevados. As características são definidas pela irreversibilidade, progressão gradual com o tempo de exposição ao risco, queda predominante nas frequências de $3000 \mathrm{~Hz}, 4000 \mathrm{~Hz}$ e $6000 \mathrm{~Hz}$, e interrupção da progressão da redução auditiva com a cessação da exposição. Tais características são decorrentes da destruição das células sensoriais do órgão de Corti, produzindo uma deterioração auditiva lenta, em geral simétrica e tendo seus aspectos clínicos relativamente definidos $(10,11)$

De acordo com a American College of Occupational and Environmental Medicine (11), o primeiro sinal de perda de audição devido à exposição ao ruído é um entalhe no audiograma em 3000, 4000 e/ou $6000 \mathrm{~Hz}$, com recuperação em $8000 \mathrm{~Hz}$. A Norma Regulamentadora $\mathrm{n}^{\mathrm{0}} 7$ do Ministério do Trabalho (12), determina que casos sugestivos de desencadeamento da PAIR podem ser verificados pela comparação do exame de referência e o sequencial, através da diferença de 10dBNA na média das frequências de 3000, 4000 e $6000 \mathrm{~Hz}$ ou piora em uma das frequências citadas em 15dBNA.

Após o rebaixamento das frequências de 3000, 4000 e $6000 \mathrm{~Hz}$, a lesão estende-se às frequências de 8000 , 2000, 1000, 500 e 250Hz. Na medida em que as frequências médias e graves são atingidas, a curva audiométrica se configura de forma descendente, havendo geralmente recuperação em $8000 \mathrm{~Hz}(13,14)$.

O ruído é o mais frequente dos agentes de exposição ocupacional, podendo desenvolver efeitos auditivos e extra-auditivos. Tais efeitos afetam diretamente a concentração e as habilidades necessárias para as tarefas gerando desatenção, descuido e mascarando sinais de alerta, contribuindo, dessa forma, para o aumento de acidentes de trabalho (8).

Os efeitos auditivos encontrados em trabalhadores portadores da PAIR limitam a funcionalidade auditiva, provocando alteração de sensibilidade auditiva, alterações na 
seletividade de frequências, na resolução temporal e espacial, recrutamento (aumento da sensação de desconforto) e zumbido. Tais alterações influenciam diretamente a discriminação auditiva, dificultando a percepção, principalmente, dos sons da fala, podendo, também, alterar o padrão de fala de acordo com o grau da perda auditiva15, 16.

Vários estudos referem à relação entre PAIR e zumbido, sendo influenciado por fatores como idade e tempo de serviço $(6,17,18)$. Um estudo demonstrou que trabalhadores com tempo médio de serviço de 6,8 anos já possuem queixa de zumbido. Tal trabalho indicou, também, a ocorrência deste sintoma em 70\% dos indivíduos com limiares normais expostos ao ruído ocupacional (18).

Com relação às alterações extra-auditivas, essas são caracterizadas por distúrbios em vários sistemas. Os distúrbios irão depender de fatores como frequência do ruído, a intensidade, a duração, e o ritmo, assim como o tempo de exposição, a suscetibilidade individual e a atitude de cada indivíduo frente ao som. As principais alterações extraauditivas encontradas em pacientes portadores de PAIR são: distúrbios de comunicação, vestibulares, comportamentais, digestivos, neurológicos, distúrbios do sono, cardiovasculares, hormonais, circulatórios, alterações nos reflexos respiratórios, na concentração e habilidade e alterações no rendimento de trabalho $(19,20)$.

Outro fator de risco que o piloto agrícola está exposto é a intoxicação por agrotóxico, através da inalação. Alguns dos compostos químicos dos agrotóxicos possuem propriedades neurotóxicas, sendo seus impactos estudados por diversas áreas da saúde, sobretudo as repercussões auditivas $(21,22)$, e considerados como responsáveis por intoxicações de trabalhadores que os manuseiam e os aplicam. As formas de intoxicação podem ser por contato com a pele, inalação e via digestiva, sendo a via respiratória a forma de absorção mais rápida (22).

Os compostos químicos mais utilizados nos agrotóxicos lançados por aviões agrícolas são os organofosforados, os piretroides sintéticos, ambos comumente usados em inseticidas, e os carbamatos, empregados em inseticidas e herbicidas (23).

Em decorrência do conhecimento dos fatores de risco ao qual o piloto agrícola está exposto, surgiu a necessidade de analisar o perfil audiológico desta classe trabalhadora, a fim de fornecer dados para pesquisas futuras.

\section{Método}

A presente pesquisa constitui-se em um estudo de coorte transversal, individual, observacional e contemporâ- neo, onde o fator em estudo são os pilotos agrícolas. O objetivo do estudo foi analisar o perfil audiológico desta população. A população foi constituída de 43 pilotos agrícolas que compareceram à inspeção de saúde no Hospital da Aeronáutica de Canoas (HACO) - Canoas/RS para a renovação do Certificado de Capacidade Física (CCF), no período de junho a setembro de 2009. Este estudo foi analisado e aprovado pelo Comitê de Ética em Pesquisa do Centro Universitário Metodista - IPA, com o protocolo número 60/2009. Sendo também aprovado pelo diretor do HACO, através da assinatura do Termo Institucional.

Foram incluídos todos os pilotos que compareceram aos exames periódicos e que aceitaram em participar deste estudo, através da assinatura do Termo de Consentimento Livre e Esclarecido. Como critérios de exclusão, foram desconsiderados os pilotos que possuíam perda auditiva condutiva. Dessa forma, foram excluídos dois aviadores, sendo a amostra composta de 41 pilotos agrícolas.

Para a coleta dos dados, os indivíduos responderam um questionário (Apêndice A) com perguntas referentes à sua rotina de trabalho e, posteriormente, foram submetidos à meatoscopia e à audiometria tonal liminar. Foram pesquisados os limiares audiométricos de via aérea das frequências de $250 \mathrm{~Hz}$ a $8000 \mathrm{~Hz}$ e de via óssea de $500 \mathrm{~Hz}$ a 4000Hz. Foi considerado padrão de normalidade limiares audiométricos iguais ou menores que 25dBNA. Para a execução da Audiometria Tonal Liminar foram utilizados dois audiômetros de dois canais, cada um, da marca GrasonStadler, modelo GSI 61, com calibração de 29/09/2008. Para a análise dos dados audiológicos por indivíduo, foi utilizada a classificação audiométrica proposta por Fiorini (24), sendo divididos em três grupos:

Grupo I: Limiares auditivos normais. Tal grupo foi subdividido em:

- Normalidade bilateral (limiares iguais ou inferiores a $25 \mathrm{~dB}$ ).

- Normalidade com entalhe unilateral (correspondendo ao rebaixamento dentro da normalidade nas frequências de 3000, 4000 e/ou 6000Hz).

- Normalidade com entalhe bilateral (idem ao anterior, porém nas duas orelhas).

A caracterização do entalhe auditivo utilizada neste trabalho é determinada, pela mesma autora (24), como o rebaixamento das frequências altas $(3000 \mathrm{~Hz}$ e/ ou $4000 \mathrm{~Hz}$ e/ou $6000 \mathrm{~Hz}$ ) de pelo menos $10 \mathrm{~dB}$ de diferença com a frequência anterior ou posterior.

Grupo II: Alteração audiométrica sugestiva de PAIR (perda auditiva nas frequências de $3000 \mathrm{~Hz}$ e/ou $4000 \mathrm{~Hz}$ e/ou 6000Hz). Subdivide-se em: 
- PAIR unilateral (apresentando audiograma sugestivo de PAIR em uma orelha e a outra com normalidade).

- PAIR unilateral com entalhe na outra orelha (traçado audiométrico sugestivo de PAIR em uma orelha e presença de entalhe dentro da normalidade na outra).

- PAIR bilateral (em ambas as orelhas).

Grupo III: traçado audiométrico sugestivo de perda descendente bilateralmente ou unilateralmente.

A análise estatística foi executada no software Statistical Package for Social Science (SPSS) 10.0 for Windows.

A análise descritiva das variáveis avião, uso de equipamentos de proteção individual (EPI), tipo de EPI utilizado, tempo de vôo diário, contato com agrotóxico, tipo de produto agrotóxico, repouso auditivo, presença sintomas auditivos e extra-auditivos, tipos de sintomas, configuração audiométrica foram descritas por meio de frequências absoluta e relativas; as variáveis idade e tempo de serviço foram analisadas por meio da observação do cálculo da média acompanhada pelo desvio padrão; e das variáveis: média dos agudos da orelha direita, média dos agudos da orelha esquerda, média dos graves da orelha direita, média dos graves da orelha esquerda foi realizada por meio da observação do cálculo de média mediana e intervalo interquartil.

A comparação da média dos agudos e graves entre as orelhas foi realizada através do teste de Wilcoxon. Para verificar a correlação entre variáveis quantitativas com distribuição normal foi utilizado o teste Qui-quadrado e para as sem distribuição normal foi usado o coeficiente de Spearman.

Todos os testes foram realizados na forma bi-caudal, admitindo-se como estatisticamente significativos os valores de $\mathrm{P}$ menores ou iguais a 0,05 .

\section{RESULTADOS}

\section{Resultados do questionário}

A população estudada foi composta por 41 pilotos agrícolas, todos do sexo masculino, com média de idade de 38,1, desvio padrão de 10,6 e faixa etária predominante de 31 a 40 anos (46,3\%). A mediana do tempo de serviço foi de 11 anos, sendo que a maioria dos indivíduos $(31,7 \%)$ são pilotos que possuem 11 a 20 anos de profissão. Quanto ao tempo de vôo diário, mais da metade $(51,2 \%)$ voam de 5 a 8 horas por dia. Os dados das variáveis encontram-se na Tabela 1.
Tabela I. Frequência das variáveis idade e tempo de serviço $(n=4 I)$.

\begin{tabular}{|c|c|c|}
\hline Variável & $\mathrm{n}$ & $\%$ \\
\hline $\begin{array}{l}\text { Idade (anos) } \\
\text { I8 a } 30 \text { anos } \\
31 \text { a } 40 \text { anos } \\
41 \text { a } 50 \text { anos } \\
51 \text { a } 60 \text { anos } \\
61 \text { a } 70 \text { anos }\end{array}$ & $\begin{array}{l}9 \\
19 \\
7 \\
3 \\
3\end{array}$ & $\begin{array}{l}22 \\
46,3 \\
17,1 \\
7,3 \\
7,3\end{array}$ \\
\hline $\begin{array}{l}\text { Tempo de serviço } \\
\text { até } 6 \text { meses } \\
7 \text { a } 11 \text { meses } \\
\text { I a } 5 \text { anos } \\
6 \text { a } 10 \text { anos } \\
\text { II a } 20 \text { anos } \\
21 \text { a } 30 \text { anos } \\
31 \text { a } 40 \text { anos }\end{array}$ & $\begin{array}{c}1 \\
2 \\
11 \\
5 \\
13 \\
6 \\
3\end{array}$ & $\begin{array}{c}2,4 \\
4,9 \\
26,8 \\
12,2 \\
31,7 \\
14,6 \\
7,3\end{array}$ \\
\hline $\begin{array}{l}\text { Tempo de vôo diário } \\
2 \text { a } 4 \text { horas } \\
5 \text { a } 8 \text { horas } \\
9 \text { a } 12 \text { horas } \\
\text { mais de } 12 \text { horas }\end{array}$ & $\begin{array}{c}2 \\
23 \\
15 \\
1\end{array}$ & $\begin{array}{c}4,9 \\
51,2 \\
36,6 \\
2,4\end{array}$ \\
\hline
\end{tabular}

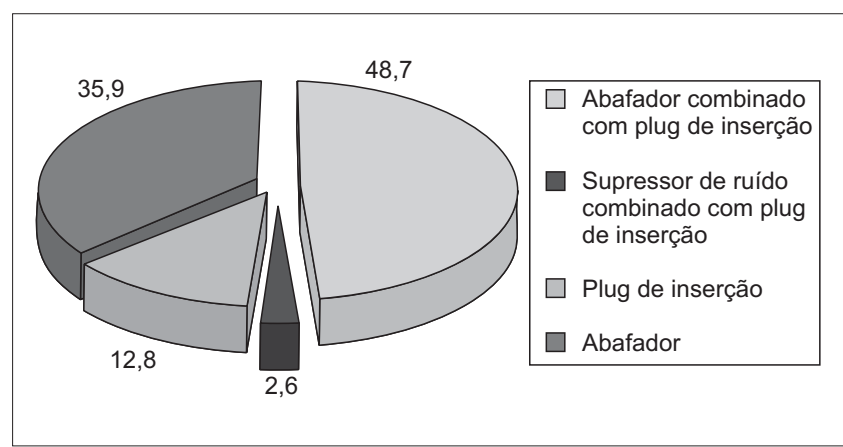

Figura 1. Distribuição do tipo de EPI utilizado pelos aviadores.

Foram pesquisados os modelos de aviões utilizados na aviação agrícola, os mais relatados foram: Ipanema Embraer 202 (43,2\%), Ipanema Embraer 201-A (41,5\%) e o Cessna AG Truck 188B (29,3\%). Os modelos Ipanema Embraer 202-A, 201, 200-A e 200, corresponderam, juntos, a $24,4 \%$.

Quanto ao uso de EPI durante os vôos, os dados revelam que $95,3 \%$ dos pilotos (39 indivíduos) utilizam tais equipamentos e 4,9\% (dois indivíduos) não o fazem. Com relação ao tipo de EPI (Figura 1), a maioria dos sujeitos $(48,7 \%)$ relatou a utilização combinada de abafador e plug de inserção. 
Mais da metade dos aviadores $(58,5 \%)$ mencionaram que possuem algum tipo de contato com produtos agrotóxicos, enquanto que $41,5 \%$ não têm qualquer contato. Dos pilotos que têm contato, $41,7 \%(n=10)$ relataram ter contato com todos os tipos de agrotóxicos (herbicida, inseticida, fungicida e maturador). O inseticida foi o produto mais citado pela amostra estudada, constituindo $95,8 \%$ das respostas, seguido do herbicida $87,5 \%$, logo após o fungicida com $83,3 \%$ e o maturador com $45,9 \%$ das respostas.

A grande maioria dos pilotos $(70,7 \%)$ alegou ter realizado mais de 14 horas de repouso auditivo antes do exame de audiometria, 12,2\% citaram ter feito de 11 a 14 horas de repouso, 9,8\% fizeram de 9 a 10 horas, 2,4\% realizaram 5 a 8 horas e 2,4\% relataram menos de 4 horas de repouso auditivo.

Foi pesquisada a presença de sintomas auditivos e extra-auditivos, afirmada por $53,7 \%$ dos pilotos $(n=22)$ e negada por $46,3 \%$, sendo o zumbido $(54,5 \%)$ o sintoma de maior prevalência entre os 22 indivíduos, e constituindo 29,3\% de respostas entre a amostra estudada (Tabela 2). Dos indivíduos que referiram queixa de zumbido $(n=12)$, 50\% pertence ao Grupo I e 50\% ao Grupo II.

\section{Resultados da avaliação audiológica}

Com relação aos dados audiológicos, a média das frequências altas $(3000,4000$ e $6000 \mathrm{~Hz})$ da orelha direita foi de $12,11 \mathrm{~dB}$ e a mediana $10 \mathrm{~dB}$ (intervalo interquartil: 5 a 18,33) e da orelha esquerda $13,05 \mathrm{~dB}$ com mediana de 8,33dB (intervalo interquartil: 5 a 22,49), a média das frequências de 500, 1000 e $2000 \mathrm{~Hz}$, em ambas as orelhas, foi de 6,66dB (OD: 3,33 a 10; OE: $2,50$ a 8,33$)$.

$\mathrm{Na}$ análise da avaliação audiométrica, foi possível verificar audição dentro dos padrões de normalidade (Grupo I) em 28 indivíduos $(68,3 \%)$ e 13 pilotos $(31,7 \%)$ com audiogramas alterados. Os dados encontram-se na Tabela 3.

Analisando os 28 audiogramas do Grupo I (100,0\%), verificamos que $17,9 \%$ de indivíduos possuem limiares normais sem entalhe, $35,7 \%$ denotam presença de entalhe unilateral e 46,4\% com presença de entalhe bilateral.

Já no Grupo II, dentre os 12 audiogramas avaliados (100,0\%), percebemos que $25 \%$ dos pilotos apresentam perda auditiva sugestiva de PAIR unilateral, $25 \%$ possuem perda auditiva unilateral com entalhe, dentro da normalidade na outra orelha e 50\% classificam-se como perda auditiva bilateral.
Tabela 2. Frequência dos sintomas auditivos e extraauditivos relatados.

\begin{tabular}{lcc}
\hline Variável & Número de respostas & $\%$ \\
\hline Sintomas Otológicos & 19 & 46,3 \\
Nenhum sintoma & 4 & 9,8 \\
Desconforto a sons fortes & 3 & 7,3 \\
Sensação de "ouvido cheio" & 4 & 9,8 \\
Diminuição da audição & I & 29,3 \\
Zumbido & 2 & 4,9 \\
Otalgia & $\mid$ & 2,4 \\
Dor de cabeça & $\mid$ & 2,4 \\
Dificuldade em entender palavras & $\mid$ & 2,4 \\
Tontura & $\mid$ & 2,4 \\
Outros &
\end{tabular}

Tabela 3. Análise da avaliação audiométrica, segundo Fiorini (1994).

\begin{tabular}{lcc}
\hline Classificação grupo & $\mathrm{n}$ & $\%$ \\
\hline Grupo I & 28 & 68,3 \\
Limiares auditivos normais bilateral & 5 & 12,2 \\
Limiares auditivos normais com entalhe unilateral & 10 & 24,4 \\
Limiares auditivos normais com entalhe bilateral & 13 & 31,7 \\
Grupo II & 12 & 29,3 \\
Perdaauditiva unilateral & 3 & 7,3 \\
Perdaauditiva unilateral com entalhe na outra orelha & 3 & 7,3 \\
Perdaauditiva bilateral & 6 & 14,7 \\
Grupo III & I & 2,4 \\
\hline
\end{tabular}

Tabela 4. Análise de pares de variáveis (Teste Qui-quadrado).

\begin{tabular}{lc}
\hline Pares de Variáveis & Valor de p \\
\hline Idadex Classificação audiométrica & 0,149 \\
Uso de EPIxClassificação audiométrica & 0,793 \\
Tempo de vôo diário x Classificação audiométrica & 0,609 \\
Contato com agrotóxico x Classificação audiométrica & 0,088 \\
\hline
\end{tabular}

* $p<0,050$ estatisticamente significante

No Grupo III foi encontrado apenas um sujeito com perda auditiva relacionada a outras causas, sendo esta unilateral.

Não encontrou-se relação estatisticamente significante nas comparações feitas das variáveis idade, uso de EPI, tempo de vôo diário e contato com agrotóxico, com a classificação audiométrica segundo Fiorini (24) (Tabela 4).

Através do teste de Wilcoxon foram comparadas as médias dos graves com a média dos agudos em cada orelha, encontrando-se diferença estatisticamente signifi- 
cativa (OD: $\mathrm{p}=0,002$; $\mathrm{OE}: \mathrm{p}<0,001)$. Tal diferença não foi identificada na comparação das frequências agudas entre as orelhas $(\mathrm{p}=0,237)$, indicando simetria das mesmas.

Na Tabela 5 estão as descritas as correlações realizadas das variáveis tempo de serviço e repouso auditivo com a média dos agudos das orelhas direita e esquerda, mostrando relação estatisticamente significante no tempo de serviço com a média dos agudos das orelhas.

Verificando a distribuição das variáveis idade, tempo de vôo diário e tipo de EPI utilizado, com a classificação dos subgrupos proposta por Fiorini (24) (Tabela 6), observase ligeira homogeneidade na variável idade e maior número de indivíduos com perda auditiva sugestiva de PAIR que pilotam de 5 a 8 horas por dia. Com relação aos EPIs

Tabela 5. Análise do Coeficiente de Spearman para pares de variáveis.

\begin{tabular}{lcr}
\hline Pares de Variáveis & Rs & Valor de $p$ \\
\hline Tempo de serviço x média agudos OD & 0,326 & $0,038^{*}$ \\
Tempo de serviço x média agudos OE & 0,399 & $0,010 *$ \\
Repouso auditivo x média agudos OD & $-0,044$ & 0,786 \\
Repouso auditivo x média agudos OE & $-0,047$ & 0,771 \\
\hline
\end{tabular}

* $p<0,050$ estatisticamente significante utilizados, em todos os tipos há presença de perda auditiva e/ou limiares auditivos normais com configuração de entalhe.

\section{DISCUSSÃO}

Este estudo teve como limitação a ausência de literatura nacional e escassez de trabalhos em nível internacional relacionados à aviação civil, principalmente na aviação agrícola, de tal forma que realizamos a maioria das comparações dos achados com a literatura relacionada à PAIR dentro de outras áreas de trabalho.

De acordo com os resultados da amostra estudada $(n=41)$, a faixa etária variou entre 25 e 66 anos, com maior número de indivíduos na faixa dos 31 a 40 anos (n=19), seguido de 18 a 30 anos ( $n=9$ ), constituindo, assim, uma população adulta jovem em plena atividade profissional. Outros estudos também mencionam possuir média e maior concentração de trabalhadores dentro desta faixa etária (3, 7). Observando a Tabela 6 , vemos que em todas as idades há presença de perda auditiva, sendo esta relativamente homogênea. Assim, podemos inferir que, na amostra estudada, a variável idade não se mostrou expressiva, já que correlacionando com a configuração audiométrica não houve relação estatística significante $(\mathrm{p}=0,149)$.

Tabela 6. Distribuição das variáveis, em percentual, relacionada com a classificação dos subgrupos proposta por Fiorini (24).

\begin{tabular}{|c|c|c|c|c|c|c|c|}
\hline Variável & $\begin{array}{l}\text { LAN } \\
\text { Bilateral }\end{array}$ & $\begin{array}{c}\text { LAN } \\
\text { c/Ent Uni }\end{array}$ & $\begin{array}{l}\text { LAN } \\
\text { c/Ent Bi }\end{array}$ & $\begin{array}{l}\text { PAIR } \\
\text { Uni }\end{array}$ & $\begin{array}{c}\text { PAIR } \\
\text { Uni+Ent }\end{array}$ & $\begin{array}{c}\text { PAIR } \\
\text { Bilateral }\end{array}$ & NãoPAIR \\
\hline \multicolumn{8}{|l|}{ Idade } \\
\hline 18 a 30 anos & 2,4 & 7,3 & 7,3 & - & 2,4 & 2,4 & - \\
\hline 31 a 40 anos & 9,8 & 12,2 & 17,1 & 2,4 & - & 4,9 & - \\
\hline 41 a 50 anos & - & 2,4 & 4,9 & 2,4 & 2,4 & 2,4 & 2,4 \\
\hline 51 a 60 anos & - & - & 2,4 & 2,4 & - & 2,4 & - \\
\hline 61 a 70 anos & - & 2,4 & - & - & 2,4 & 2,4 & - \\
\hline \multicolumn{8}{|l|}{ Tempo de vôo diário } \\
\hline 2 a 4 horas & - & 2,4 & 2,4 & - & - & - & - \\
\hline 5 a 8 horas & 9,8 & 12,2 & 12,2 & 7,3 & 4,9 & 9,8 & - \\
\hline 9 a 12 horas & 2,4 & 9,8 & 14,6 & - & 2,4 & 4,9 & 2,4 \\
\hline Mais de 12 horas & - & - & 2,4 & - & - & - & - \\
\hline \multicolumn{8}{|l|}{ Tipo de EPI } \\
\hline Abafador & - & 10,3 & 10,3 & 2,6 & 2,6 & 7,7 & 2,6 \\
\hline Plug & 5,1 & 2,6 & 5,1 & - & - & - & - \\
\hline Abafador + plug & 7,7 & 10,3 & 15,4 & 5,1 & 5,1 & 5,1 & - \\
\hline Supressor ruído + plug & $g \quad-$ & - & 2,6 & - & - & - & - \\
\hline
\end{tabular}

Legenda: LAN - Limiares Auditivos Normais; Ent Uni - Entalhe Unilateral; Ent Bi - Entalhe Bilateral; PAIR - Sugestiva de Perda Auditiva Induzida pelo Ruído; PAIR Uni + Ent - Sugestiva de Perda Auditiva Induzida pelo Ruído Unilateral com Entalhe na outra orelha; Não PAIR - Perda auditiva não sugestiva de Perda Auditiva Induzida pelo Ruído. 
O tempo de serviço demonstrou que $26,8 \%$ dos aviadores $(\mathrm{n}=11)$ trabalham na aviação agrícola de 1 (um) a 5 anos e 12,2\% pilotam de 6 a 10 anos, considerados como um curto espaço de tempo de exposição. Porém, trabalhadores que possuem até 10 anos de exposição poderão apresentar perda auditiva instalada em um estágio inicial, mas com danos irreversíveis (14). O maior agrupamento de indivíduos $(31,7 \%)$ é de pilotos agrícolas entre 11 a 20 anos de profissão, sendo um tempo considerável de exposição. Foi possível verificar dependência estatística significante entre o tempo de serviço com a média dos agudos (OD: $\mathrm{p}=0,030 ; \mathrm{OE}: \mathrm{p}=0,010)$, indicando que pilotos com maior tempo de profissão terão a média das frequências agudas de maior valor. Esse achado também foi encontrado nos estudos de Lopes et al (18), e condiz com a história natural da PAIR quanto ao agravamento da mesma, se mantida a exposição ao ruído (14).

Um fator preocupante na aviação agrícola é o tempo de exposição diária ao ruído, onde verificamos que $36 \%$ dos pilotos $(\mathrm{n}=15)$ voam de 9 a 12 horas por dia e $56,1 \%$ (23 indivíduos) trabalham diariamente de 5 a 8 horas. Embora não tenha sido encontrada relação estatística significante entre o tempo de vôo diário com a configuração audiométrica $(\mathrm{p}=0,609)$, na Tabela 6 podemos verificar que as perdas auditivas concentram-se na faixa de tempo de exposição entre 5 e 12 horas diárias. Percebemos que em todos os grupos há presença de entalhe tanto unilateral quanto bilateral mostrando que o ruído pode afetar a audição mesmo com tempo mínimo de exposição. Um dado curioso é que o grupo que mencionou voar de 5 a 8 horas por dia é mais afetado pelo ruído do que o grupo que pilota entre 9 a 12 horas. Porém, este dado pode ser explicado pelo fato que, em tempos de safra, o número de horas de vôo por dia aumenta, uma vez que os prazos para término das aplicações são curtos e é neste período que o piloto agrícola possui maior demanda de trabalho. Assim, evidenciamos a importância de verificar as horas de vôo neste período. Tal fator é preocupante, pois não há consenso legal sobre os limites diários/ mensais de vôo na aviação agrícola. A lei que vigora sobre todos os aeronautas é a Lei 7.183/84 (25), que estabelece que uma tripulação simples pode voar 9 horas e 30 minutos por dia, com máximo de 11 horas de jornada de trabalho.

Quanto aos aviões, os três modelos mais utilizados (Ipanema Embraer 202, Ipanema Embraer 201-A e Cessna AG Truck 188B), são aviões monomotores, com média de 300 cavalos de potência (HP). O avião Ipanema Embraer 201-A e os modelos anteriores a ele (modelos 201, 200-A e 200), possuem seu escapamento nas laterais frontais, direcionados à cabine do piloto. O ruído gerado acaba por atingir diretamente o cockpitem intensidades elevadas. Os modelos Ipanema Embraer 202, 202-A e Cessna AG Truck 188B possuem seu escapamento na parte inferior do avião evitando que o ruído, produzido pelo mesmo, alcance diretamente a cabine do aviador (26). KIEFER et al (23), fizeram uma análise de dois aviões agrícolas Rockwell Thrush SR2, sendo monomotores de 600HP. O monitoramento de ruído destes aviões demonstrou que o nível equivalente de ruído $\left(\mathrm{L}_{\text {eq }}\right)$ ficou entre $103 \mathrm{~dB}(\mathrm{~A})$ e $104 \mathrm{~dB}(\mathrm{~A})$. Tais valores ultrapassam os limites de tolerância fixados pela Norma Regulamentadora ${ }^{\circ} 15$ do Ministério do Trabalho (27), que define $85 \mathrm{~dB}$ para 8 horas de trabalho. Assim, fica clara a importância da avaliação do nível de ruído destes aviões, a fim de proporcionar valores reais para cada avião e, a partir disso, pensar em propostas de prevenção.

O Regulamento Brasileiro de Homologação Aeronáutica (RBHA) 137 (28) estabelece que qualquer operação aeroagrícola não será realizada sem que os tripulantes utilizem máscara para respiração com filtro contra inalação de produtos tóxicos e capacete anti-choque com dispositivos para fixação de viseiras e abafadores de ruído. O uso de EPI para a audição foi confirmado por $95,1 \%$ dos pilotos $(n=39)$, embora 4,9\% $(n=2)$ não sigam a determinação legal. Tal lei menciona apenas o uso de abafadores, mas mais da metade da população $(51,2 \%)$ utiliza o uso combinado de abafador e plug de inserção, sendo uma informação positiva quanto ao interesse da prevenção de perdas auditivas. No entanto, mesmo com esta medida, as perdas auditivas mantêm-se presentes (Tabela 6). Isto ocorreu também com o uso isolado do abafador, que aparentemente se mostrou menos eficaz do que a medida anterior. Em todos os tipos de EPI foi vista a presença de entalhe, indicando que a atenuação pode não ser suficiente para o nível de ruído e/ou o tempo de exposição pode estar contribuindo para o seu aparecimento. KIEFER et al (23) estudou o uso de capacete para vôo, tendo o nível de redução de ruído (NRR) de 8,3dB. Comparado ao nível de ruído, concluiu que os capacetes, isoladamente, não oferecem proteção suficiente à exposição durante os vôos.

Com relação aos agrotóxicos, 58,5\% dos pilotos mencionaram ter contato com esses produtos, um número considerável de indivíduos que estão expostos aos seus efeitos nocivos. Os inseticidas seguido dos herbicidas, foram os agrotóxicos mais citados pela população, dado semelhante ao encontrado por outros estudos $(21,22)$. De acordo com Monquero et al. (22), as intoxicações por agrotóxicos podem ser agudas ou crônicas. Na primeira, os sintomas são dores de cabeça, tonturas, diarreia, fraqueza, perturbação da visão, dores de estômago, sonolência, salivação e/ou suor excessivos e dificuldades respiratórias. Já na crônica, os efeitos podem surgir meses ou anos após a exposição. Testamos a correlação entre o contato com o agrotóxico e a classificação audiométrica, não sendo encontrada relação estatística significante. Contudo, verificamos que há uma tendência estatística, já que o valor do p 
$(\mathrm{p}=0,088)$ ficou próximo ao nível de significância $(\mathrm{p}=0,050)$. Por isso, ressaltamos a necessidade de uma investigação mais aprofundada acerca dos efeitos dos produtos agrotóxicos à audição desta população, uma vez que a exposição aos agentes ototóxicos é preocupante.

A Portaria no 19/98 (9) define que até a realização do exame de audiometria, o trabalhador deverá permanecer em repouso auditivo por no mínimo 14 horas, o que foi seguido por $70,7 \%$ dos pilotos $(n=29)$. Como $29,3 \%$ dos aviadores não fizeram o devido repouso auditivo, verificamos se a média dos agudos foi influenciada pelo tempo do repouso (Tabela 5), não sendo encontrada relação estatística significante (OD: $p=0,786$; OE: $p=0,771$ ).

O zumbido foi o sintoma otológico de maior referência pesquisada entre os indivíduos que possuíam tais sintomas, correspondendo a $54,5 \%$ das repostas. A prevalência do zumbido relacionada ao número total de pilotos equivale a $29,3 \%$, sendo o valor encontrado maior do que o estudo feito por Steinmetz et al. (18), que correspondeu a $22 \%$. A literatura coloca que o zumbido é o primeiro sinal de alerta de uma exposição a sons de níveis de pressão sonora elevados, podendo ser sintoma de mudança temporária do limiar (10). Para tanto, dos 12 aviadores que referiram queixa de zumbido, metade possuem limiares auditivos dentro da normalidade (quatro com configuração de entalhe bilateral e dois com entalhe unilateral) e metade com perda auditiva sugestiva de PAIR.

A diferença estatística significativa entre média de agudos e graves entre ambas as orelhas (OD: $\mathrm{p}=0,002$; OE: $\mathrm{p}<0,001)$, juntamente com a constatação de simetria da média das frequências agudas das mesmas $(p=0,237)$, constituem quadro compatível com a PAIR, por possuir característica de queda predominante nas frequências de $3000 \mathrm{~Hz}, 4000 \mathrm{~Hz}$ e $6000 \mathrm{~Hz}$ e alteração auditiva simétrica $(9,10,20)$.

Na classificação das audiometrias conforme Fiorini (24) verificamos que 29,3\% dos pilotos agrícolas possuem perda auditiva sugestiva de PAIR, número maior que vários estudos em outras áreas ocupacionais, porém próximo aos resultados encontrados em pilotos de aeroclube (3) que apresentaram 30\% de perda auditiva sugestiva de PAIR. Dentro dos audiogramas sugestivos de PAIR ( $n=12)$, metade dos indivíduos possuem perda auditiva bilateral. Encontramos normalidade em $68,3 \%$ dos aviadores, no entanto, o dado mais surpreendente é o número de audiogramas com configuração de entalhe, representando $24,4 \%$ com entalhe unilateral e $31,7 \%$ com entalhe bilateral, totalizando $56,1 \%$ da amostra estudada. Pela história natural da PAIR, sabe-se que estes pilotos tendem a desenvolver perda auditiva com a continuidade da exposição $(11,14)$. Somando os valores dos audiogramas sugestivos de PAIR com os audiogramas com configuração de entalhe, temos um total de $85,4 \%$ de pilotos agrícolas que são afetados pelos efeitos do ruído sobre a audição. Este é um número extremamente relevante e que deve ser considerado no desenvolvimento de políticas de prevenção da PAIR.

\section{CONCLUSÃO}

Com este estudo podemos concluir que os aviadores agrícolas estão realmente expostos aos efeitos ocasionados pelo ruído. Tal atividade ocupacional envolve contato com produtos agrotóxicos, tempo de exposição ao ruído e ainda o nível do próprio ruído. Embora haja o uso de EPI, as perdas auditivas apareceram na amostra estudada. A prova disso está na ocorrência elevada de perdas auditivas sugestivas de PAIR e, ainda, pelo aparecimento de configuração de entalhe em mais da metade da população.

Pudemos constatar que a idade não foi estatisticamente significante quando comparada com a classificação da audiometria, porém quanto maior o tempo de serviço, maior o comprometimento das frequências agudas. O zumbido foi o sintoma de maior prevalência, afetando indivíduos com perda auditiva e com audição normal.

Este é um estudo inicial dentro da aviação agrícola. Esperamos que, com este trabalho, haja um maior direcionamento das equipes de saúde e segurança do trabalho e das autoridades competentes para a saúde do piloto agrícola, seja na prevenção das perdas auditivas e dos demais riscos que esta população está exposta, seja na promoção de saúde. Para isso, são necessários novos estudos e, talvez, repensar a própria legislação deste tipo de aviação.

\section{REFERÊNCIAS BIBLIOGRÁFICAS}

1. Silveira VR. Cenário atual da aviação agrícola no Brasil. São José dos Campos, 2004, p. 184, (Tese de Mestrado Instituto Tecnológico de Aeronáutica).

2. Antuñano MJ, Spanyers JP. Hearing and noise in aviation. FAA CAMI. Oklahoma City, 1998. Publicação: AM-400-98/ 3.

3. Gerostergiou E, Tsitiridis I, Batzakakis D, Limpanovnou G, Vathilakis I, Sandris V. Sensorineural hearing loss of noise in members of aviation club of Larissa (Greece). Hippokratia. 2008, 12(1):50-63.

4. Linden M.B. Os conteúdos da consciência do trabalhador exposto ao ruído. São Paulo, 1996, p. 147, (Dissertação de Mestrado - Pontifícia Universidade Católica de São Paulo). 
5. Lopes G, Russo ICP, Fiorini AC. Estudo da audição e da qualidade de vida em motoristas de caminhão. Rev Cefac. 2007, 9(1):532-42.

6. Dias A, Cordeiro R, Conente JE, Gonçalves CGO. Associação entre perda auditiva induzida pelo ruído e zumbidos. Cad de Saúde Pública. 2006, 22(1):63-68.

7. Santos JD, Ferreira MIDC. Variação dos limiares audiométricos em trabalhadores submetidos a ruído ocupacional. Arq Int Otorrinolaringol. 2008, 12(2):201-09.

8. Gonçalves, CGO. Saúde do Trabalhador: da estruturação à avaliação de programas de preservação auditiva. São Paulo: Roca; 2009.

9. Brasil. Ministério do Trabalho. Portaria no 19 GM/SSSTb, de 9 de abril de 1998. Estabelece diretrizes e parâmetros mínimos para avaliação e acompanhamento da audição em trabalhadores expostos a níveis de pressão sonora elevados. DOU, São Paulo. 22 abril. 1998.

10. Nudelmann AA, Costa EA, Seligman J, Ibañez RN (org). PAIR - Perda Auditiva Induzida pelo Ruído. Rio de Janeiro: Revinter; 2001.

11. American College of Occupational and Environmental Medicine; Acoeme Evidence Based Statement. Noise induced hearing loss. J Occup Environ Med. 2003, 45.

12. Brasil. Ministério do Trabalho. Secretária da Segurança e do Trabalho. Norma Regulamentadora nำ 7. Programa de Controle Médico de Saúde Ocupacional. DOU, Brasília. 30 dez. 1994.

13. Maniglia JV, Carmo KC. Avaliação dos danos por ruídos em trabalhadores de marcenaria. Acta Orl. 1998, 7(2):9096.

14. Almeida SIC, Albernaz PLM, Zaia P, Xavier OG, Karazava EHI. História natural da perda auditiva ocupacional provocada por ruído. Rev Ass Med Bras. 2000, 46(2):14348.

15. Samelli AG. Zumbido: avaliação, diagnóstico e reabilitação: abordagens atuais. São Paulo: Lovise; 2004.

16. Mello A. Alerta ao Ruído Ocupacional. Porto Alegre, 1999, p. 74, (Monografia da Especialização em Audiologia Clínica - Centro de Especialização em Fonoaudiologia Clínica/RS).

17. Lopes AC, Nelli MP, Lauris JRP, Amorim RB, Melo ADP. Condições de saúde auditiva no trabalho: investigação dos efeitos auditivos em trabalhadores expostos ao ruído ocupacional. Arq Int Otorrinolaringol. 2009, 13(1):49-54.
18. Steinmetz LG, Zeigelboim BS, Lacerda AB, Morata TC, Marques JM. Características do zumbido em trabalhadores expostos a ruído. Rev Bras Otorrinolaringol. 2009, 75(1):714.

19. Medeiros LB. Ruído: Efeitos extra-auditivos no corpo humano. Porto Alegre, 1999, p. 33, (Monografia da Especialização em Audiologia Clínica - Centro de Especialização em Fonoaudiologia Clínica/RS).

20. Ferreira Junior M. PAIR - Perda Auditiva Induzida por Ruído - Bom Senso e Consenso. 1. ed. São Paulo: Editora VK; 1998.

21. Castro, JSM, Confalonieri U. Uso de Agrotóxicos no Município de Cachoeiras de Macau (RJ). Ciênc Saúde Coletiva. 2005, 10(2):473-82.

22. Monquero PA, Inácio EM, Silva AC. Levantamento de agrotóxicos e utilização de equipamento de proteção individual entre os agricultores da região de Araras. Arq Inst Biol. 2008, 76(1):135-39.

23. Kiefer M, Lenhart SW, Tubbs RL, \& Mattorano D. HHE Report No. HETA-95-0248-2562, Dirty Bird, Inc., Grady, Arkansas. NIOSH, Cincinnati, Ohio, 1996. Disponível em: http://www.cdc.gov/niosh/hhe/reports/pdfs/1995-02482562.pdf

24. Fiorini AC. Conservação auditiva: estudo sobre o monitoramento audiométrico em trabalhadores de uma indústria metalúrgica. São Paulo, 1994, p. 98, (Dissertação de Mestrado - Pontifícia Universidade Católica de São Paulo).

25. BRASIL, Lei no 7.183/84. Regula o exercício da profissão de aeronauta e dá outras providências. DOU, Brasília. 05 de abril. 1984.

26. Brasil. Comando da Aeronáutica. Anac. Especificações de Tipo de Produtos Aeronáuticos noㅡ EA - 7104. Brasília, 2005. Disponível em: http://www.anac.gov.br/certificacao/ Produtos/Espec/EA-7104-07p.pdf

27. Brasil. Ministério do Trabalho. Norma Regulamentadora 15. Limites de tolerância para ruído contínuo ou intermitente. Portaria nº 3.214. DOU, Brasília. 8 jun. 1978.

28. Brasil. Comando da Aeronáutica. Anac. Portaria No 454 DGAC /STE - Regulamento Brasileiro de Homologação Aeronáutica 137: Operações Aeroagrícolas. DOU, Brasília. 08 de jul.1999. Disponível em: http://www.anac.gov.br/ biblioteca/rbha/rbha137.pdf. 